\title{
Isospin phases of vertically coupled double quantum rings under the influence of perpendicular magnetic fields
}

\author{
M. Royo, ${ }^{1}$ F. Malet, ${ }^{2, *}$ M. Barranco, ${ }^{2}$ M. Pi, ${ }^{2}$ and J. Planelles ${ }^{1, \dagger}$ \\ ${ }^{1}$ Departament de Química Física i Analítica, Universitat Jaume I, Box 224, E-12080 Castelló, Spain \\ ${ }^{2}$ Departament ECM and IN2UB, Facultat de Física, Universitat de Barcelona, Diagonal 647, 08028 Barcelona, Spain
}

(Received 30 July 2008; published 7 October 2008)

\begin{abstract}
Vertically coupled double quantum rings submitted to a perpendicular magnetic field $B$ are addressed within the local spin-density-functional theory. We describe the structure of quantum ring molecules containing up to 40 electrons considering different inter-ring distances and intensities of the applied magnetic field. When the rings are quantum mechanically strongly coupled, only bonding states are occupied and the addition spectrum of the artificial molecules resembles that of a single-quantum ring, with some small differences appearing as an effect of the magnetic field. Despite the latter's tendency to flatten the spectra, in the strong-coupling limit, some clear peaks are still found even when $B \neq 0$ that can be interpretated from the single-particle energy levels similarly as in the zero magnetic field case, namely, in terms of closed-shell and Hund's-rule configurations. By increasing the inter-ring distance, the occupation of the first antibonding orbitals washes out such structures and the addition spectra become flatter and irregular. In the weak-coupling regime, numerous isospin oscillations are found as functions of $B$.
\end{abstract}

DOI: 10.1103/PhysRevB.78.165308

PACS number(s): 73.21.-b, 85.35.Be, 71.15.Mb, 75.75.+a

\section{INTRODUCTION}

Systems made of correlated electrons confined in semiconductor nanoscopic dot and ring structures, the so-called quantum dots (QDs) and rings (QRs), respectively, have been the subject of intense theoretical and experimental researches (see, e.g., Refs. 1 and 2 and references therein). From the latter point of view, for quantum dots, it has been proved $^{3}$ the possibility to tune over a wide range the number of electrons contained in the system, as well as to control both the size and the shape of the dots by means of external gate voltages - a goal that has not been achieved yet for ring geometries due to the higher complexity of their fabrication process, ${ }^{4-6}$ which involves several experimental techniques such as atomic force microscopy, ${ }^{7}$ strain-induced self-organization, ${ }^{4}$ and droplet molecular-beam epitaxy. ${ }^{8}$

The interest of QRs arises from their peculiar behavior in the presence of a perpendicularly applied magnetic field $(B)$, which is very distinct from that observed in QDs and shows up as an oscillatory behavior of their energy levels as a function of $B$. This property, together with the fact that in narrow enough QRs the electrons experiment a nearly onedimensional Coulomb repulsion, leads to the integer and fractional Aharonov-Bohm effects usually associated with the appearance of the so-called persistent currents in the ring. ${ }^{9}$ These quantum-interference phenomena have been experimentally reported ${ }^{10}$ and have motivated a series of theoretical works whose number is steadily increasing (see, e.g., Refs. 11-16 and references therein).

One of the most appealing possibilities offered by electron systems confined in semiconductor heterostructures is their ability to form coupled entities, usually referred to as "artificial molecules," in which the role of the constituent "atoms" is played by single-quantum dots or rings and that have analogies with natural molecules such as the hybridization of the electronic states forming molecularlike orbitals. In addition, these artificially coupled systems present important advantages such as a tunable "interatomic" coupling by means of, e.g., the modification of the relative position/size of the constituents. This fact has, besides its intrinsic interest, potential relevance to quantum information processing schemes since basic quantum gate operations require controllable coupling between qubits. In this sense, artificial molecules based on two coupled QDs called quantum dot molecules (QDMs) have been proposed as scalable implementations for quantum computation purposes and have received great attention from the scientific community in the last years (see, e.g., Refs. 17-26 and references therein).

Also, molecular-beam epitaxy techniques have recently allowed the synthesis of quantum ring molecules (QRMs) in the form of concentric double QRs (Refs. 27 and 28) and vertically stacked layers of self-assembled QRs..$^{29,30}$ The optical and structural properties of the latter have also been characterized by photoluminescence spectroscopy and by atomic force microscopy, respectively. This has sparked theoretical studies on the structure and optical response of both vertically and concentrically coupled QRs of different complexity and scope, revealing properties different from those of their dot counterparts due to the nonsimply connected ring topology. For instance, studies on the single-electron spectrum of vertical QRMs (Refs. 31 and 32) have shown that the electronic structure of these systems is more sensitive to the inter-ring distance than that of coupled QDs. As a consequence, in ring molecules, quantum tunneling effects are enhanced since less tunneling energy is required to enter the molecularlike phase. Also, the consideration of "heteronuclear" artificial molecules constituted by slightly different QRs offers the interesting possibility to control the effective coupling of direct-indirect excitons ${ }^{33}$ by means of the application of a magnetic field and taking advantage of the fact that charge tunneling between states with distinct angular momentum is strongly suppressed by orbital selection rules. To this end, some authors have considered the case of QRMs 
made of strictly one-dimensional zero-thickness QRs and have used diagonalization techniques to address the fewelectron problem. ${ }^{31,33-35}$ The simultaneous effect of both electric and magnetic fields applied to a single-electron QRM has also been studied ${ }^{36}$ (see also Ref. 31), and the optical response of QRMs where the thickness of the constituent QRs is taken into account has been obtained. ${ }^{37}$ In addition, the spatial correlation between electron pairs in vertically stacked QRs has been shown to undergo oscillations as functions of the magnetic flux, with strongly correlated situations between ground states (gs's) with odd angular momentum turning out to occur even at large inter-ring distances. ${ }^{34}$ More recently, the structure of a QRM made of two vertically stacked quantum rings has been addressed at zero magnetic field for a few tens of electrons within the local spin-density-functional theory (LSDFT) neglecting ${ }^{38}$ and incorporating ${ }^{39}$ the vertical thickness of the constituent QRs.

In this work we address the gs of two thick vertically coupled identical quantum rings forming "homonuclear" QRMs populated with up to 40 electrons and pierced by a perpendicularly applied magnetic field. We extend in this way our previous study, ${ }^{39}$ addressing the appearance and physical interplay between the spin and isospin ${ }^{23}$ degrees of freedom as functions of the variation in both the intensity of the magnetic field and the inter-ring separation. Modeling systems charged with such large number of electrons requires the employment of methodologies that minimizes the computational cost. Here we have made use of the LSDFT, ${ }^{13,15}$ whose accuracy for the considered values of the magnetic field has been assessed ${ }^{24}$ by comparing the obtained results for a single QD with those given by the current-spin-density-functional theory, ${ }^{40}$ which is in principle better suited for high magnetic fields and also with exact results for artificial molecules. ${ }^{41}$

This paper is organized as follows. In Sec. II we briefly introduce the LSDFT and the model used to represent the vertical QRMs. In Sec. III we discuss the obtained results for some selected configurations and a summary is given in Sec. IV.

\section{DENSITY-FUNCTIONAL CALCULATION FOR MANY- ELECTRON VERTICAL QUANTUM RING HOMONUCLEAR MOLECULES}

The axial symmetry of the system allows one to work in cylindrical coordinates. The confining potential $V_{\mathrm{cf}}(r, z)$ has been taken parabolic in the $x y$ plane with a repulsive core around the origin, plus a symmetric double quantum well in the $z$ direction, each one with width $w$, depth $V_{0}$, and separated by a distance $d$. To improve on the convergence of the calculations, the double-well profile has been slightly rounded off as illustrated in Fig. 2 of Ref. 24. The potential thus reads $V_{\mathrm{cf}}(r, z)=V_{r}(r)+V_{z}(z)$, where

$$
V_{r}(r)=V_{0} \Theta\left(R_{0}-r\right)+\frac{1}{2} m \omega_{0}^{2}\left(r-R_{0}\right)^{2} \Theta\left(r-R_{0}\right),
$$

$$
V_{z}(z)=V_{0} \begin{cases}\frac{1}{1+e^{(z+d / 2+w) / \sigma}}-\frac{1}{1+e^{(z+d / 2) / \sigma}} & \text { if } z \leq 0 \\ \frac{1}{1+e^{(z-d / 2) / \sigma}}-\frac{1}{1+e^{(z-d / 2-w) / \sigma}} & \text { if } z>0,\end{cases}
$$

with $\sigma=2 \times 10^{-3} \mathrm{~nm}$ and $\Theta(x)=1$ if $x>0$ and zero otherwise. The convenience of using a hard-wall confining potential to describe the effect of the inner core in QRs is endorsed by several works in the literature. ${ }^{42}$ We have taken $R_{0}$ $=10 \mathrm{~nm}, V_{0}=350 \mathrm{meV}, \hbar \omega_{0}=6 \mathrm{meV}$, and $w=5 \mathrm{~nm}$. These parameters determine the confinement for the electrons together with the distance between the constituent quantum wells that is varied to study QRMs in different inter-ring coupling regimes.

For small electron numbers $(N)$, it is justified to take $\omega_{0}$ to be $N$ independent. However, in a more realistic scheme its value should be tuned according to the number of electrons contained in the system, relaxing the confinement as the latter is increased. In the case of quantum dots it has often been used a $N^{-1 / 4}$ dependence that arises from the $r$ expansion near the origin of the Coulomb potential created by a twodimensional uniform positive charge distribution (jellium model) and that it is generalized to the case of quantum dot molecules as $\omega_{0}=\kappa N_{B}^{-1 / 4}$ with $N_{B}$ being the number of electrons filling bonding (B) orbitals (see below). The rationale for this generalization is given in Ref. 25. It is clear that the mentioned $N$ dependence would be harder to justify for QRs, and in fact no alternative law is known for a single QR that could be generalized to the case of QRMs. For this reason, in this work, we have taken $\omega_{0}$ to be $N$ independent, which is to some extent less realistic for the largest values of $N$ we have considered.

Considering the $N$-electron system placed in a magnetic field parallel to the $z$ axis, within LSDFT in the effective mass, dielectric constant approximation, the Kohn-Sham equations ${ }^{24,43}$ in cylindrical coordinates read

$$
\begin{gathered}
{\left[-\frac{1}{2}\left(\frac{\partial^{2}}{\partial r^{2}}+\frac{1}{r} \frac{\partial}{\partial r}-\frac{l^{2}}{r^{2}}+\frac{\partial^{2}}{\partial z^{2}}\right)-\frac{\omega_{c}}{2} l+\frac{1}{8} \omega_{c}^{2} r^{2}+V_{\mathrm{cf}}(r, z)+V_{H}\right.} \\
\left.+V_{x c}+\left(W_{x c}+\frac{1}{2} g^{*} \mu_{B} B\right) \eta_{\sigma}\right] u_{n l \sigma}(r, z)=\varepsilon_{n l \sigma} u_{n l \sigma}(r, z),
\end{gathered}
$$

where the single-particle (sp) wave functions have been taken to be of the form $\phi_{n l \sigma}(r, z, \theta, \sigma)=u_{n l \sigma}(r, z) e^{-l l \theta} \chi_{\sigma}$, where $n=0,1,2, \ldots, l=0, \pm 1, \pm 2, \ldots$ with $-l$ being the projection of the single-particle orbital angular momentum on the symmetry axis, and $\sigma=\uparrow(\downarrow)$ representing spin-up (-down) states. The vector potential has been chosen in the symmetric gauge, namely, $\mathbf{A}=B(-y, x, 0) / 2 ; \mu_{B}=\hbar e /\left(2 m_{e} c\right)$ and $\omega_{c}=e B / c$ are, respectively, the Bohr magneton and the cyclotron frequency and $\eta_{\sigma}=+1 \quad(-1)$ for $\sigma=\uparrow(\downarrow) ; V_{H}(r, z)$ is the direct Coulomb potential and $V_{x c}=\partial \mathcal{E}_{x c}(n, m) /\left.\partial n\right|_{\mathrm{gs}}$ and $W_{x c}=\partial \mathcal{E}_{x c}(n, m) /\left.\partial m\right|_{\mathrm{gs}}$ are the variations in the exchangecorrelation energy density $\mathcal{E}_{x c}(n, m)$ in terms of the electron density $n(r, z)$ and of the local spin magnetization $m(r, z)$ 


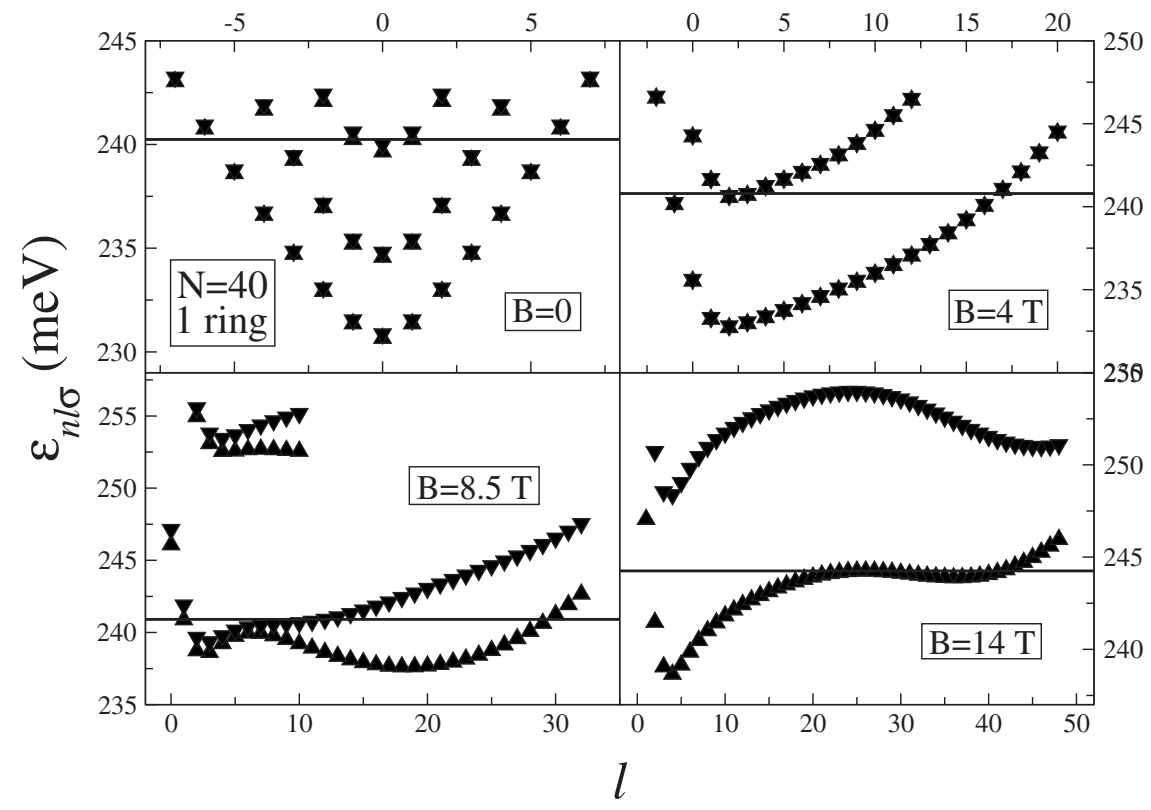

FIG. 1. Single-electron energies $(\mathrm{meV})$ as functions of $l$ for a $N=40$ single ring. Upward (downward) triangles denote $\uparrow(\downarrow)$ spin states. The horizontal lines represent the Fermi levels. The value of $B(\mathrm{~T})$ is indicated in each panel.

$\equiv n^{\uparrow}(r, z)-n^{\downarrow}(r, z)$ taken at the gs. $\mathcal{E}_{x c}(n, m) \equiv \mathcal{E}_{x}(n, m)$ $+\mathcal{E}_{c}(n, m)$ has been built from three-dimensional homogeneous electron gas calculations; this yields a well-known ${ }^{44}$ simple analytical expression for the exchange contribution $\mathcal{E}_{x}(n, m)$. For the correlation term $\mathcal{E}_{c}(n, m)$ we have used the parametrization proposed by Perdew and Zunger. ${ }^{45}$ Details about how the Kohn-Sham and the Poisson equations have been solved can be found in Ref. 43. Notice the use of effective atomic units $\hbar=e^{2} / \epsilon=m=1$ in Eq. (2), where $\epsilon$ is the dielectric constant and $m$ is the electron effective mass. In units of the bare electron mass $m_{e}$ one has $m=m^{*} m_{e}$ with the length unit being the effective Bohr radius $a_{0}^{*}=a_{0} \epsilon / m^{*}$ and the energy unit the effective Hartree $H^{*}=H^{*} / \epsilon^{2}$. In the numerical applications we have considered GaAs quantum rings, for which we have taken $\epsilon=12.4$ and $m^{*}=0.067$; this yields $a_{0}^{*} \sim 97.9 \AA$ and $H^{*} \sim 11.9 \mathrm{meV}$ with the effective gyromagnetic constant $g^{*}=-0.44$.

To label the gs configurations ("phases") we use an adapted version of the ordinary spectroscopy notation, ${ }^{41}$ namely, ${ }^{2 S+1} L_{g, u}^{ \pm}$, where $S$ and $L$ are the total $\left|S_{z}\right|$ and $\left|L_{z}\right|$, respectively. The superscript $+(-)$ corresponds to symmetric (antisymmetric) states under reflection with respect to the $z=0$ plane bisecting the QRMs and the subscript $g(u)$ refers to positive (negative) parity states. All of these are good quantum numbers even in the presence of an axial magnetic field. By analogy with natural molecules, symmetric and antisymmetric states are referred to as $B$ and antibonding $(\mathrm{AB})$ orbitals, respectively. We have defined the "isospin" quantum number $I_{z}$ (bond order in Molecular Physics) as ${ }^{22,24,41} I_{z}$ $=\left(N_{\mathrm{B}}-N_{\mathrm{AB}}\right) / 2$ with $N_{\mathrm{B}(\mathrm{AB})}$ being the number of occupied bonding (antibonding) sp states.

\section{RESULTS}

Due to the large number of variables needed to characterize a given QRM configuration (electron number, magnetic field, and inter-ring distance), we limit ourselves to present results in a limited range of values for such variables, aiming at discussing calculations that might illustrate the appearance of some properties of the systems under study. For the sake of comparison, we have also addressed one single QR symmetrically located with respect to the $z=0$ plane with the same thickness $(5 \mathrm{~nm})$ and radial confinement as the coupled rings.

Figure 1 shows the Kohn-Sham sp levels for one single ring hosting $N=40$ electrons as a function of $l$ for different values of the applied magnetic field. As it is well known, these levels are $\pm l$ degenerate at $B=0$. In this particular case, the gs has $S_{z}=1$, and it is made up of symmetric (with respect to $z=0)$ sp states with up to $n=3$. In the noninteracting single-electron model, in which the Coulomb energy is not considered and consequently the $\mathrm{sp}$ wave functions factorize into a $r$-dependent and a $z$-dependent part with associated quantum numbers $n_{r}$ and $n_{z}$, i.e., $u_{n l}(r, z) \rightarrow \mathcal{U}_{n_{r}}(r) \mathcal{Z}_{n_{z}}(z)$, one would say that the gs is made up of sp states with $n_{z}=0$ and radial quantum numbers up to $n_{r}=3$.

When $B \neq 0$, the $\pm l$ degeneracy is lifted and, on the other hand, the $l<0$ sp levels become progressively depopulated in favor of those with $l>0$ as the magnetic field increases until eventually (at about $\sim 4 \mathrm{~T}$ ) only $l>0$ orbitals are filled. At this point, only a few states with $n=2$ are occupied and the ring has $S_{z}=0$. From this value of $B$, the simultaneous filling of increasingly higher- $l$ states and those close to $l=0$ gives rise to configurations containing only states with $n=1$ and with large values of the total spin (e.g., $S_{z}=9$ for $B$ $=8 \mathrm{~T})$. Eventually, the system becomes fully spin polarized at $B \sim 13.5 \mathrm{~T}$. It is worth noticing the conspicuous bending of the "Landau bands" (sets of bonding or antibonding states characterized by the same $n$ and spin and different value of $l$ ) instead of displaying a fairly flat region, as it happens when the in-plane confinement is produced by a jelliumlike potential $^{13}$ but not with our present choice of a $N$-independent parabola. It is also worth to stress that, due to the much stronger confinement in the vertical direction as compared to that in the radial one, only symmetric states are occupied. 


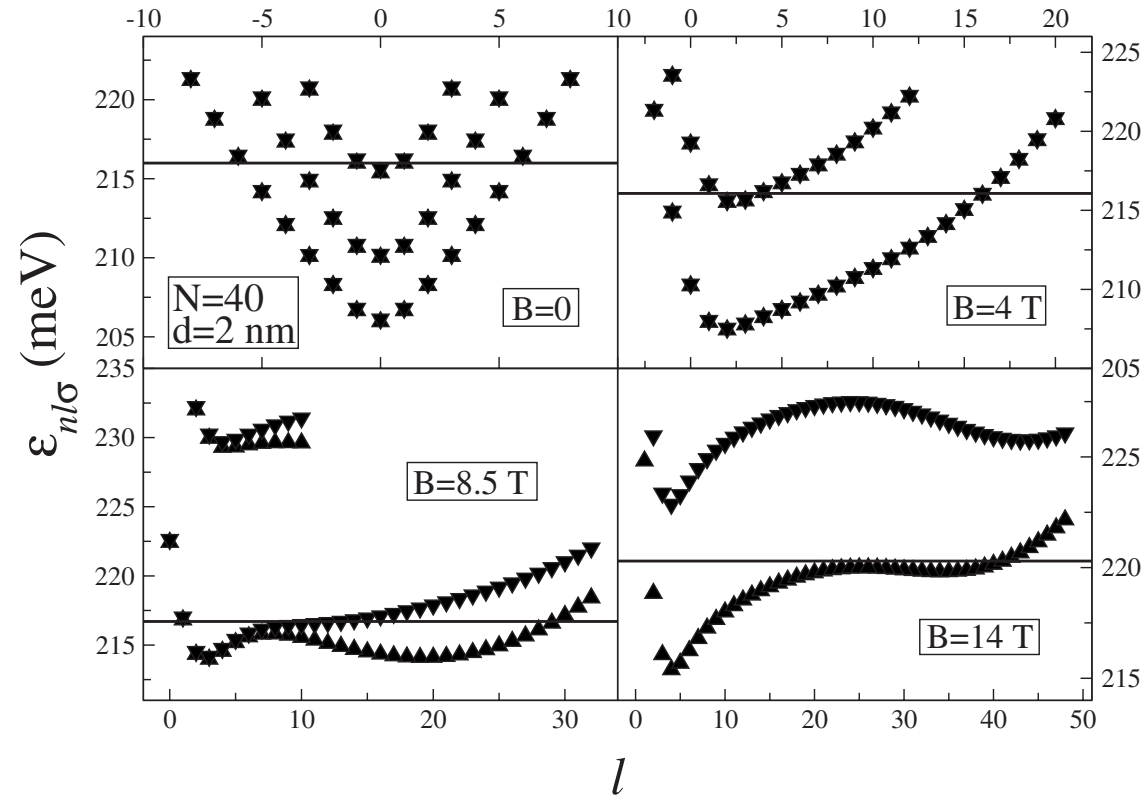

FIG. 2. Same as Fig. 1 for a QRM with $N=40$ electrons and $d$ $=2 \mathrm{~nm}$. Notice that due to the small separation between the rings only bonding states are occupied. The antibonding ones are lying at much higher energies.

Analogously, the energy levels corresponding to QRMs with $N=40$ and inter-ring distances $d=2,4$, and $6 \mathrm{~nm}$ are shown in Figs. 2-4. One can see the gradual evolution of the system as $d$ increases; indeed, at $d=2 \mathrm{~nm}$ the spectrum is very similar to that of the single ring with only bonding $\mathrm{sp}$ states being occupied. As $d$ increases, a few antibonding orbitals become populated at small $B$ 's, as one can see from the top panels of Fig. 3, corresponding to $d=4 \mathrm{~nm}$; but eventually, for increasing values of $B$, the QRMs have again ground states where only bonding states are populated as can be seen from the bottom panels of the same figure. For this inter-ring distance, the fully spin-polarized state is reached at $B$ $\sim 13.75$ T. Finally, for the largest ring separation considered, namely, $d=6 \mathrm{~nm}$, a large amount of antibonding orbitals becomes occupied giving rise to small $I_{z}$ 's instead of the fairly large isospin values found for similar configurations at smaller distances (compare the bottom panels in Fig. 4 with those in Figs. 2 and 3). In particular, the fully spin-polarized gs is found at about $B \sim 7$ T with $I_{z}=2$, whereas for $d=2$ and $4 \mathrm{~nm}$ it appears near $B=14 \mathrm{~T}$ and has the maximum possible isospin value, namely, $I_{z}=20$. At $d=6 \mathrm{~nm}$, the maximumspin state naturally consists of two distinct bands: one made up of bonding and another of antibonding states. These configurations are the QRM analogs of the maximum density droplet (MDD) configurations found for QDMs at similar inter-dot distances called, respectively, $\mathrm{MDD}_{\mathrm{B}}$ and $\mathrm{MDD}_{\mathrm{AB}}$ in Ref. 25. Increasing further the magnetic field causes the progressive occupation of higher- $l$ orbitals, which provokes the depopulation of the antibonding band and the consequent increase in $I_{z}$. For the highest considered magnetic field ( $B$ $\sim 14 \mathrm{~T}$ ), some antibonding orbitals are still occupied yielding $I_{z}=17$.

These results are a consequence of the evolution with $d$ of the energy difference between bonding and antibonding

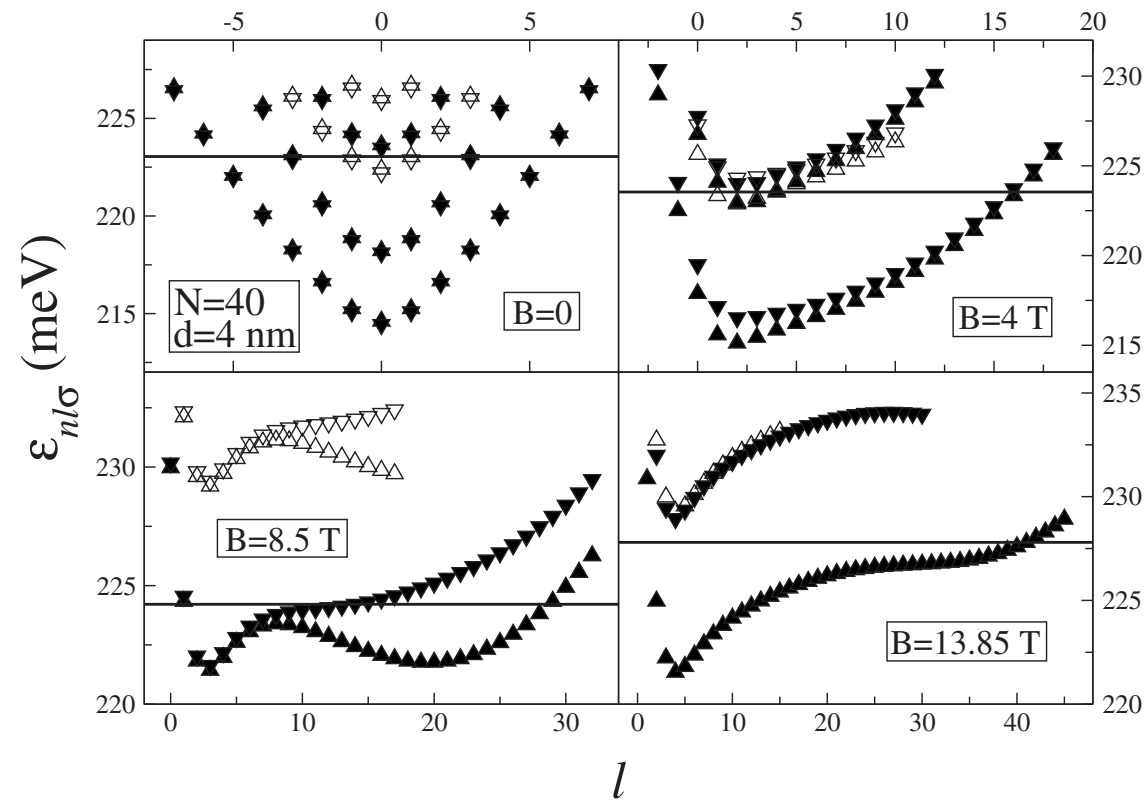

FIG. 3. Same as Fig. 2 for $d$ $=4 \mathrm{~nm}$. The increase in the interring separation allows for the occupation of both bonding and antibonding states indicated by solid and open triangles, respectively. 


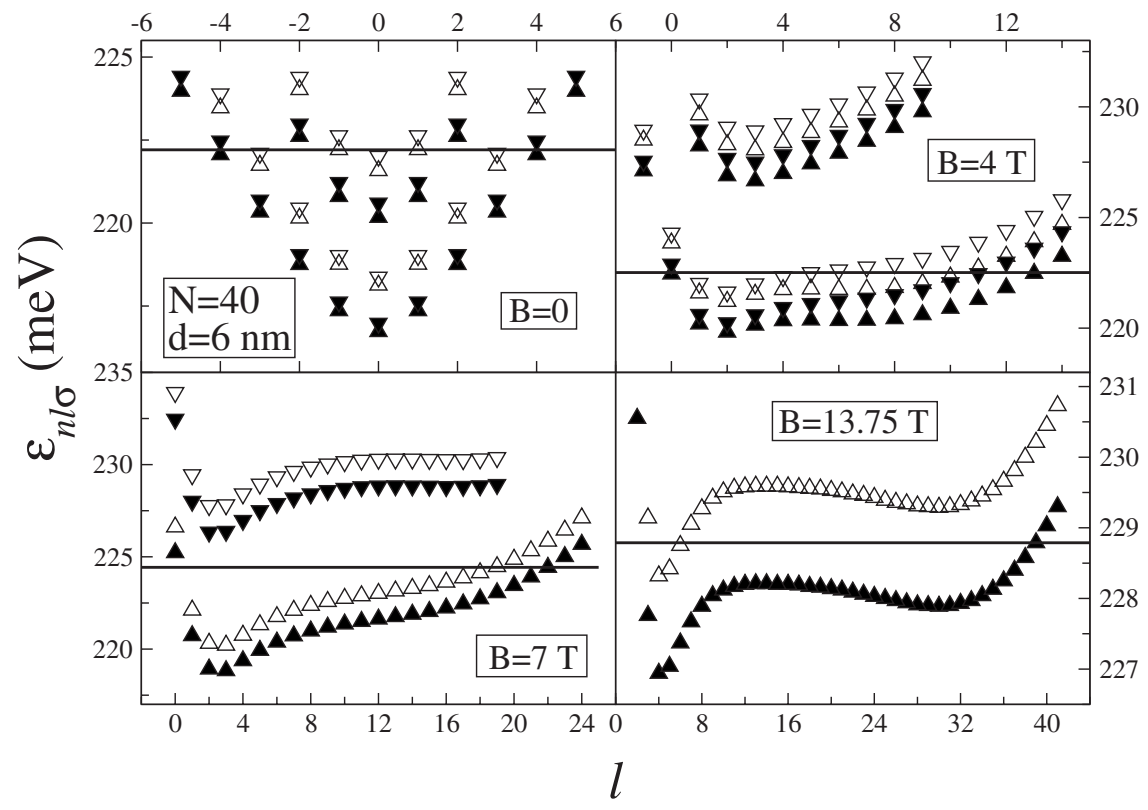

FIG. 4. Same as Fig. 3 for $d$ $=6 \mathrm{~nm}$.

states $\Delta_{\text {SAS }}$, which accurately varies as a function of the inter-ring distance according to the law $\Delta_{\mathrm{SAS}}=\Delta_{0} e^{-d / d_{0}}$ already found for QDMs. ${ }^{22}$ In our case, from the difference in energy of single-electron (bonding and antibonding) QRMs we have obtained $\Delta_{0}=82 \mathrm{meV}$ and $d_{0}=1.68 \mathrm{~nm},{ }^{39}$ values which have turned out to be unaffected by the applied magnetic field. Clearly, the value of $\hbar \omega_{0}$ as compared to $\Delta_{\mathrm{SAS}}$, which allows discerning between the strong $\left(\hbar \omega_{0} \lesssim \Delta_{\text {SAS }}\right)$ and the weak $\left(\hbar \omega_{0} \gg \Delta_{\text {SAS }}\right)$ quantum-mechanical coupling regimes, has a crucial influence on the actual filling of bonding and antibonding sp states at a given inter-ring distance. Indeed, increasing $\omega_{0}$ while keeping the double-well structure constant may favor the population of antibonding orbitals for large enough values of $N{ }^{24}$ This can be understood from the noninteracting electron model, in which the singleelectron energies are the sum of two independent terms: one arising from the $z$ localization and characterized by the quantum number $n_{z}$ and another, which increases as $\omega_{0}$ does, arising from the $r$ localization and depending on $l$ and the radial quantum number $n_{r}$. If $N$ is large enough, the QRMs can minimize their energy by populating antibonding states with low values of $n_{r}$ and $l$ instead of going on populating bonding states with higher quantum numbers. This explains why some antibonding states were filled even for $d=2 \mathrm{~nm}$ at $B=0$ and $N=40$ for the QRMs of Ref. 39, where $\omega_{0}$ was taken to be $15 \mathrm{meV}$, a value almost three times larger than the one considered in the present work. ${ }^{24}$

This particular structure of the bonding and antibonding bands at high magnetic fields may have some observable effects on the far-infrared response of QRMs. Indeed, since the dipole operator cannot connect bonding with antibonding sp states, for QRMs in the weak-coupling limit, one would expect the dipole spectrum to display additional fragmentation in the characteristic edge modes of the ring geometry ${ }^{13}$ due to the contribution of the antibonding electron-hole pairs (see, e.g., the bottom panels of Fig. 4).

Figure 5 shows the evolution with $d$ of the gs energy and the molecular phase of QRMs made up of $N=8$ electrons and submitted to magnetic fields of different intensities. Notice that even moderate values of $B$ give rise to ground states with large total angular momentum, which increases as the magnetic field does. For this reason, we have denoted it by its actual value instead of employing the usual notation with upper Greek letters except for the cases with $L_{z}=0$. Similar conclusions can be drawn for all the values of the magnetic field we have considered; on the one hand, for the studied inter-ring distances, the energy of the molecular phases increases with $d$ due to the enhancement of the energy of the bonding states, ${ }^{43}$ which dominates over the decrease in the Coulomb energy - for larger distances the constituent QRs

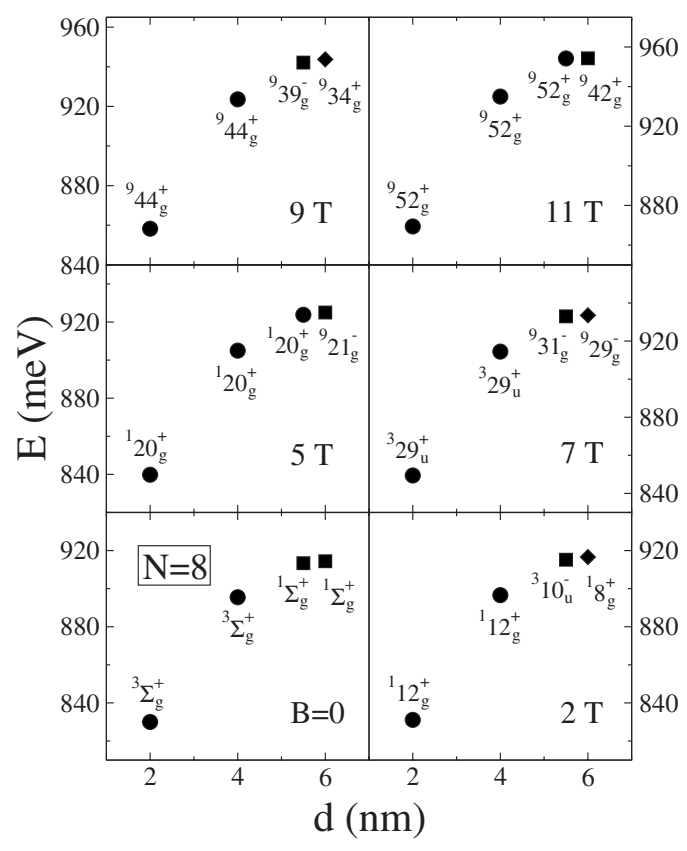

FIG. 5. Energy (meV) and gs molecular phases of the QRM with $N=8$ electrons as functions of the inter-ring distance for different values of $B$. For a given $B$ value, different phases are represented by different symbols. 


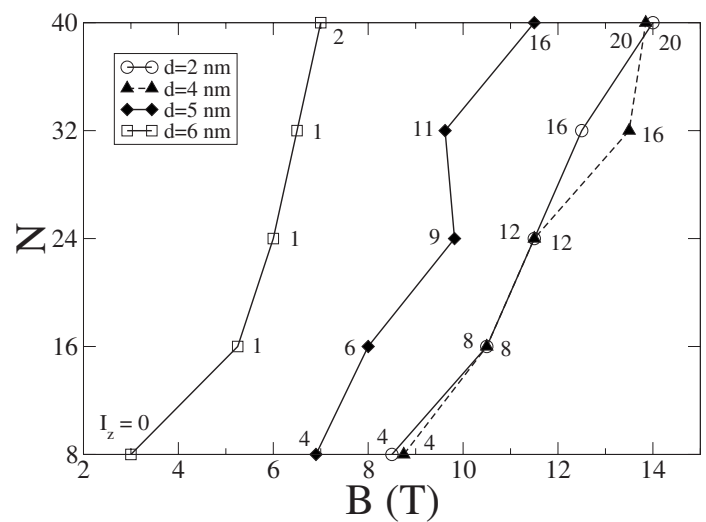

FIG. 6. $B$ values at which the selected QRMs become fully spin polarized for the inter-ring distances $d=2,4$, and $6 \mathrm{~nm}$. The isospin of each configuration is also indicated. The lines have been drawn to guide the eyes.

are so apart that eventually this decrease dominates and the tendency is reversed. On the other hand, one can see that the first phase transitions are always found at the largest interring distances since, as happens for QDMs in the fewelectron limit, they are due to the replacement of an occupied bonding sp state with an empty antibonding one. This also explains why in most of the cases, and especially for the highest magnetic fields, the total angular momentum of the QRMs in the weakest coupling regime is reduced. The filled antibonding orbitals have lower $l$ 's than the replaced bonding states.

We have determined the magnetic field that gives rise to ring molecules with fully spin-polarized gs and show it in Fig. 6 as a function of $N$ for different inter-ring distances going from the strong to the weak quantum-mechanical coupling regimes. The isospin value of each configuration is also indicated. The number of electrons, $N=8 \times M$ with $M$ $=1-5$, was chosen with the aim of producing closed-shell structures at $B=0$ in the weak-coupling limit. One can see that the results for $d=2$ and $4 \mathrm{~nm}$ are very close with only noticeable differences for $N=32$. This can be understood from the bottom panels of Figs. 2 and 3, which show that for rather large magnetic fields only bonding orbitals are occupied for both ring separations. Contrarily, from Fig. 4 one can see that in weaker coupling regimes the filling of antibonding states favors the fully spin-polarization of the QRMs at low $B$ intensities as compared to those needed when the rings are closer to each other, which explains the differentiated results corresponding to $d=5$ and $6 \mathrm{~nm}$ in Fig. 6.

When antibonding orbitals are populated, the variation in the magnetic field yields numerous transitions between different molecular phases with different isospin that are more complex than these observed in vertically coupled QDs. This particular behavior is mainly due to the periodic destabilization suffered by the lowest- $l$ occupied orbitals induced by the magnetic field, which is a direct consequence of the Aharonov-Bohm effect, and makes it rather difficult to find a pattern among the observed evolutions for the different electronic populations. The spin and isospin phases as functions of the magnetic field are shown in Fig. 7 for $d=6 \mathrm{~nm}$ cor-

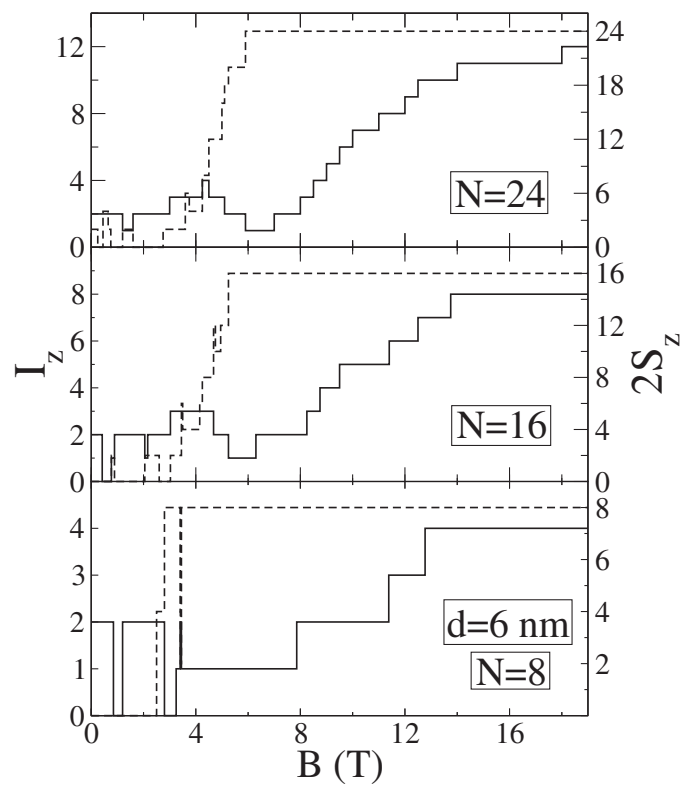

FIG. 7. Isospin (solid line, left scale) and spin (dotted line, right scale) values as functions of $B$ for the QRMs with $d=6 \mathrm{~nm}$ and $N=8,16$, and 24 .

responding to $N=8,16$, and 24 . It can be seen that in all cases at $B=0$ the QRMs have $I_{z}=2$ and $S_{z}=1$; when $B$ is increased, nonmonotonic spin and isospin oscillations with $\Delta I_{z}= \pm 1$ and $\Delta S_{z}= \pm 1$ and 2 appear, respectively. Two facts, also present in QDMs, ${ }^{24,25}$ are worth to be stressed: on one hand, molecular phase changes from $-(+)$ to $+(-)$ ground states (recall that, as explained in Sec. II, this sign is related to the symmetry of the molecular configuration) involve $\Delta I_{z}=+1(-1)$ flips; on the other hand, quite often the transitions in both magnitudes take place simultaneously except obviously when the QRMs reach the full spinpolarization point, from which on the isospin increases in one-unit jumps until the system is made up of only bonding states.

The comparison of the isospin phases for QRMs with $d$ $=4$ and $6 \mathrm{~nm}$ is presented in Fig. 8 for $N=32$ and 40. Clearly, the highest values of $I_{z}$ appear for the smallest inter-ring distances, as expected from the single-particle levels shown

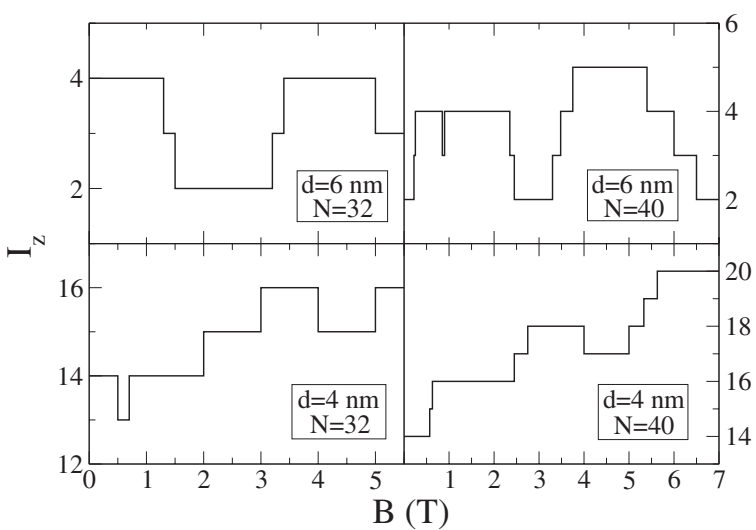

FIG. 8. Isospin values as functions of $B$ for the QRMs with $d$ $=4$ and $6 \mathrm{~nm}$, and $N=32$ and 40 . 


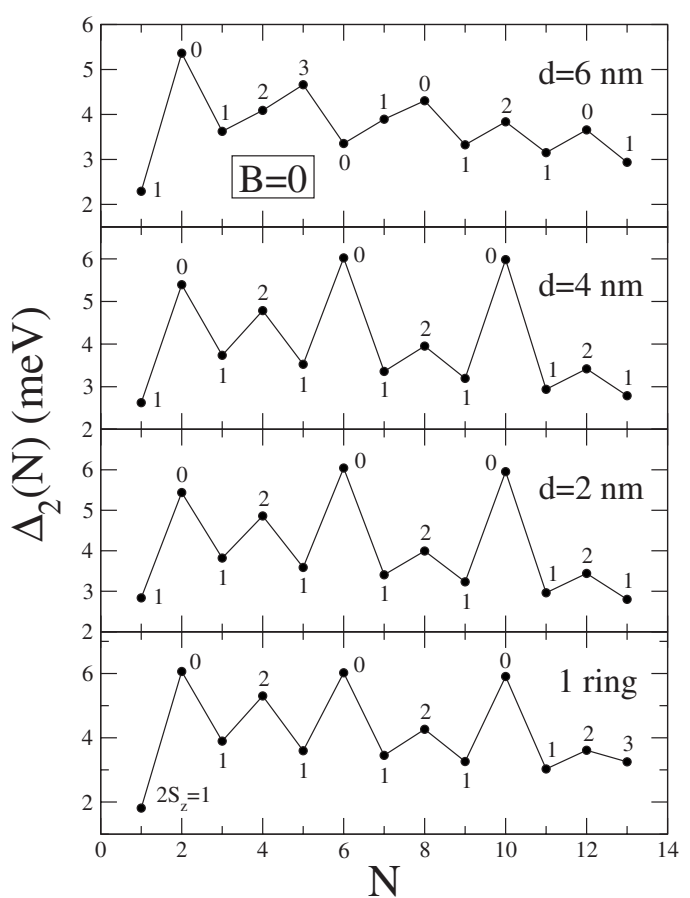

FIG. 9. Addition energies for QRMs at $B=0$ and inter-ring distances $d=2,4$, and $6 \mathrm{~nm}$ and for a single QR (bottom panel). The value of $2 S_{z}$ corresponding to each $N$ is also indicated.

in Fig. 3, corresponding to $d=4 \mathrm{~nm}$ and $N=40$, in which only a few antibonding orbitals are occupied for low values of $B$. Indeed, one can see from the bottom panels of Fig. 8 that, for this inter-ring distance, magnetic fields of about $5 \mathrm{~T}$ are enough to yield configurations with the maximum isospin value $N / 2$, whereas for the QRMs with $d=6 \mathrm{~nm}$ such values of $B$ still correspond to small $I_{z}$ 's due to the large amount of filled antibonding states.

We have also calculated the addition energies defined by

$$
\Delta_{2}(N)=E(N+1)-2 E(N)+E(N-1),
$$

with $E(N)$ being the total energy of the $N$-electron system for QRMs made of up to 14 electrons at different inter-ring distances submitted to several magnetic fields as functions of $N$. For the sake of comparison, we have also calculated $\Delta_{2}(N)$ for the corresponding single rings. The results for $B=0,3$, and $6 \mathrm{~T}$ are shown in Figs. 9-11, respectively, in which the bottom panels correspond to the single ring.

From Fig. 9 one can see that at zero magnetic field the single-QR addition spectrum presents the usual intense peaks at $N=2,6$, and 10 with zero total spin and those at $N=4$ and 8 with $S_{z}=1$ satisfying the Hund's rule. Similar results are found for the QRMs with $d=2$ and $4 \mathrm{~nm}$, indicating that such systems behave as a single ring owing to the strong quantum-mechanical coupling corresponding to these interring distances (notice that, except for $N=13$, the spin values coincide for all the configurations). This fact contrasts with the results found for the vertical ring molecules of Ref. 39, where at $d=4 \mathrm{~nm}$ the spectrum clearly reflected an intermediate coupling situation due to the filling of the first antibonding orbitals. As commented before, for the systems studied in the present paper such states are only occupied for

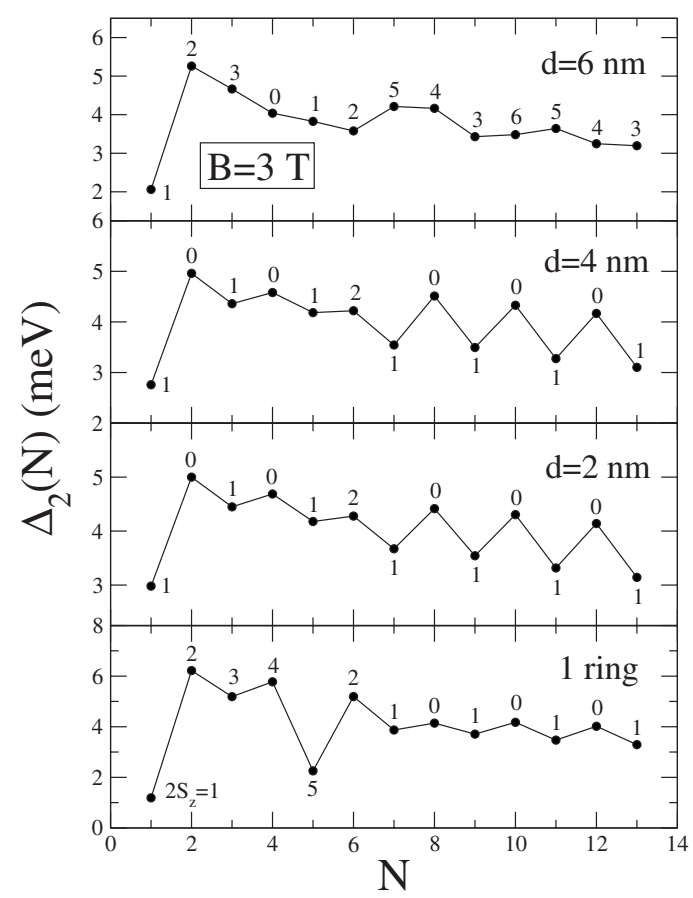

FIG. 10. Same as Fig. 9 for $B=3$ T.

larger inter-ring separations (or $N$ 's of the order of 30).

The spectrum corresponding to $d=6 \mathrm{~nm}$ is shown in the top panel of the same figure. One can see that, although some of the marked peaks are preserved, in particular those at $N$ $=2$ and 8, the ones at $N=4$ and 6 no longer exist. Notice that for 6 electrons the spectrum presents now a minimum and also a new peak is found at $N=5$. This intricate structure can be understood from the corresponding single-particle energy levels. Indeed, it appears that the QRMs with $N \leq 4$ are made up of only bonding states with the first antibonding state being filled when $N=5$. From $N \geq 7$, the QRMs have always occupied both $\mathrm{B}$ and $\mathrm{AB}$ orbitals but, however, the intermediate six-electron configuration has again only symmetric states. This alternate behavior evidences that $6 \mathrm{~nm}$ is not a separation large enough for the QRMs to be in the weakcoupling limit but rather corresponds to an intermediate regime. Notice also that, from the results of Ref. 39, in the weak-coupling limit one would expect to find clearly marked peaks at the same $N$ values as for the single ring multiplied by 2 , i.e., at $N=4,12$, and 20 , indicating that the rings are so apart that they behave as isolated entities. We have checked that, for our QRMs to present such spectrum, we should consider inter-ring distances of about $10 \mathrm{~nm}$. The different spin values for $d=6 \mathrm{~nm}$ as compared to those in the strongcoupling regime can also be explained from the sp levels. For example, the $2 S_{z}=3$ assignation of the QRM with $N=5$ is due to the above-mentioned filling of an antibonding (spin up with $l=0$ ) orbital replacing the spin-down $|l|=1$ state occupied for $d=2$ and $4 \mathrm{~nm}$. Analogously, the configuration with $S_{z}=1$ (instead of $S_{z}=0$ ) for $N=10$ can also be explained from the sp levels. In the strong-coupling limit, the QRM is formed by the spin-degenerated sp levels with $l=0,|1|$, and $|2|$, but this closed-shell configuration is prevented by the filling of the antisymmetric orbitals at $d=6 \mathrm{~nm}$. Finally, the reverse situation occurs at $N=8$, where the closing of the 
antibonding $l=0$ and $|1|$ shells contrasts with the Hund's-rule configurations found for the strongly coupled molecules.

Figure 10 shows the addition energies corresponding to the situation in which a magnetic field of $3 \mathrm{~T}$ is applied to the rings. Like what is found at $B=0$, the spectrum of the single system and of the molecules with $d=2$ and $4 \mathrm{~nm}$ are rather similar. Notice the different energy scales with the most remarkable difference being the salient minimum that appears for the single $\mathrm{QR}$ at $N=5$. For the above-mentioned interring distances, peaks with $S_{z}=0$ are found at $N=2,4,8,10$, and 12 , as well as a peak at $N=6$ with $S_{z}=2$, although they are not as clearly marked as at $B=0$. It turns out that, even in the presence of a magnetic field, when the single-particle energy levels no longer display the $\pm l$ degeneration, the QRMs can adopt configurations that are somehow analog to these characteristics of the situation at $B=0$, namely, the closed-shell ones and those fulfilling the Hund's rule. Indeed, for, e.g., $d=4 \mathrm{~nm}$ and $N=10$, the ring molecule is made up of the spin-degenerate bonding states with $l=0-4$ (instead of those with $|l|=0-2$ of the $B=0$ case). Similarly, at $N=6$ the occupied orbitals are the spin-up and -down ones with $l=1$ and 2, and the spin-up ones with $l=0$ and 3 (instead of the spin-degenerate states with $|l|=0$ and 1 filled at zero magnetic field). For larger inter-ring separations, the occupancy of the first antibonding orbitals washes out these structures and the addition spectrum becomes flatter and irregular. One can notice also the different spin assignations between the single and the coupled systems especially for the lowestpopulated configurations. In particular, the single QRs with $N \leq 5$ turn out to be fully spin polarized, which can be attributed to the combined effect of the magnetic field and a relatively strong exchange-correlation interaction characteristic of few-electron single-quantum rings. The relatively higher spin values at $d=6 \mathrm{~nm}$ for $N \geq 7$ are due to the filling of the antibonding states.

Finally, the addition energies for $B=6 \mathrm{~T}$ are shown in Fig. 11. It can be seen that in all cases the only clearly marked peak is the one at $N=2$, with the rest of the spectra being rather flat, following the trend observed at $B=3 \mathrm{~T}$. Nevertheless, some weak peaks are still found and can be interpretated as in the previous cases, e.g., the one at $N=8$ for $d=4 \mathrm{~nm}$ with $2 S_{z}=2$. The system fills the spin-up and -down states with $l=2-4$ and the spin-up ones with $l=1$ and 5. One can also notice that the faint peak of the four-electron configurations of both the single ring and the QRM with $d$ $=2 \mathrm{~nm}$ becomes a minimum at larger inter-ring distances. Concerning the spin, the single QRs and the QRMs with $d$ $=2$ and $4 \mathrm{~nm}$ turn out to be fully polarized for $N \leq 7,5$, and 3 , respectively, whereas the filling of the antibonding states favors the fully spin polarization of molecules with the largest ring separation for all the considered electron numbers.

\section{SUMMARY}

Within the local spin-density-functional theory, we have addressed the ground state of quantum ring molecules containing up to 40 electrons, with different inter-ring distances, submitted to perpendicular magnetic fields. In the strongcoupling regime the energy levels and the addition energies

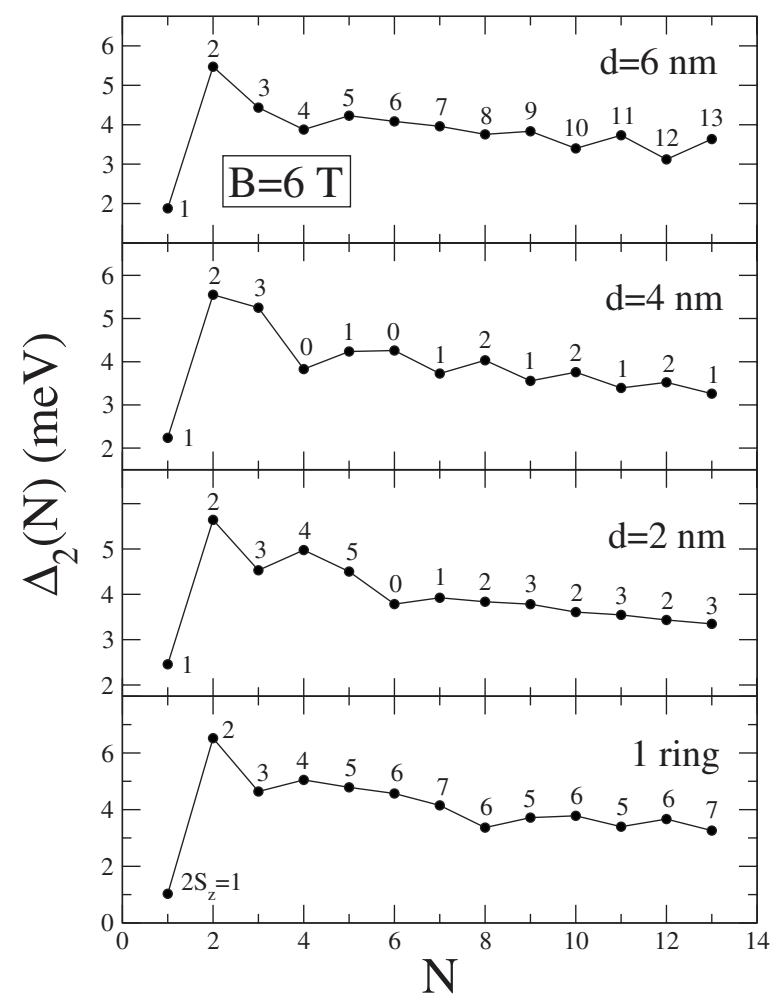

FIG. 11. Same as Fig. 9 for $B=6$ T.

of the QRMs are similar to those of a single QR, although some differences are found due to the effect of the magnetic field, which has a tendency to wash out the clearly marked peaks characteristic of the $B=0$ case as well as to yield flatter addition spectra. However, even at $B \neq 0$, some peaks are still present and they can be interpretated as in the zero magnetic field case.

When the ring separation is increased until the first antibonding orbitals are occupied, the addition spectra become irregular and the ring molecules are fully spin polarized at relatively low magnetic fields. The filling of such states yields isospin oscillations as functions of $B$ increasing in one-unit jumps once the corresponding molecular configurations reach the maximum spin value.

Despite the lack of experimental results to compare ours with, we believe that the ones herewith presented may be helpful in the analysis of future experiments on vertically coupled QRs concerning, e.g., the realization of singleelectron transistor measurements, where the evolution of the chemical potential $\mu(N)$ with the magnetic field can be experimentally identified as the variation in the position of the current peaks as a function of the applied field, showing irregularities arising from phase transitions.

\section{ACKNOWLEDGMENTS}

This work was performed under Grant No. FIS200501414 from DGI (Spain) and Generalitat Valenciana FPI (M.R.) and under Projects UJI-Bancaixa No. P1-1B2006-03 (Spain) and No. 2005SGR00343 from Generalitat de Catalunya. 
*fmalet@ecm.ub.es

†planelle@exp.uji.es

${ }^{1}$ L. Jacak, P. Hawrylak, and A. Wjs, Quantum Dots (Springer, Berlin, 1998); T. Chakraborty, Quantum Dots (Elsevier, Amsterdam, 1999); D. Bimberg, M. Grundmann, and N. N. Ledentsov, Quantum Dot Heterostructures (Wiley, London, 2001).

${ }^{2}$ E. Lipparini, Modern Many-Particle Physics (World Scientific, Singapore, 2008).

${ }^{3}$ S. Tarucha, D. G. Austing, T. Honda, R. J. van der Hage, and L. P. Kouwenhoven, Phys. Rev. Lett. 77, 3613 (1996).

${ }^{4}$ J. M. García, G. Medeiros-Ribeiro, K. Schmidt, T. Ngo, J. L. Feng, A. Lorke, J. Kotthaus, and P. M. Petroff, Appl. Phys. Lett. 71, 2014 (1997).

${ }^{5}$ A. Lorke, R. J. Luyken, A. O. Govorov, J. P. Kotthaus, J. M. Garcia, and P. M. Petroff, Phys. Rev. Lett. 84, 2223 (2000).

${ }^{6}$ A. Fuhrer, S. Lüscher, T. Ihn, T. Heinzel, K. Ensslin, W. Wegscheider, and M. Bichler, Nature (London) 413, 822 (2001); T. Ihn, A. Fuhrer, T. Heinzel, K. Ensslin, W. Wegscheider, and M. Bichler, Physica E (Amsterdam) 16, 83 (2003).

${ }^{7}$ R. Held, S. Lüscher, T. Heinzel, K. Ensslin, and W. Wegscheider, Appl. Phys. Lett. 75, 1134 (1999).

${ }^{8}$ Z. Gong, Z. C. Niu, S. S. Huang, Z. D. Fang, B. Q. Sun, and J. B. Xia, Appl. Phys. Lett. 87, 093116 (2005).

${ }^{9}$ N. A. J. M. Kleemans, I. M. A. Bominaar-Silkens, V. M. Fomin, V. N. Gladilin, D. Granados, A. G. Taboada, J. M. García, P. Offermans, U. Zeitler, P. C. M. Christianen, J. C. Maan, J. T. Devreese, and P. M. Koenraad, Phys. Rev. Lett. 99, 146808 (2007).

${ }^{10}$ T. Ihn, A. Fuhrer, K. Ensslin, W. Wegscheider, and M. Bichler, Physica E (Amsterdam) 26, 225 (2005)

${ }^{11}$ T. Chakraborty and P. Pietiläinen, Phys. Rev. B 50, 8460 (1994).

${ }^{12}$ I. V. Krive, R. I. Shekhter, S. M. Girvin, and M. Jonson, Phys. Scr., T t54, 123 (1994).

${ }^{13}$ A. Emperador, M. Barranco, E. Lipparini, M. Pi, and Ll. Serra, Phys. Rev. B 59, 15301 (1999).

${ }^{14}$ J. C. Lin and G. Y. Guo, Phys. Rev. B 65, 035304 (2001).

${ }^{15}$ M. Aichinger, S. A. Chin, E. Krotscheck, and E. Räsänen, Phys. Rev. B 73, 195310 (2006).

${ }^{16}$ Y. M. Liu, G. M. Huang, and T. Y. Shi, Phys. Rev. B 77, 115311 (2008).

${ }^{17}$ R. H. Blick, R. J. Haug, J. Weis, D. Pfannkuche, K. v. Klitzing, and K. Eberl, Phys. Rev. B 53, 7899 (1996).

${ }^{18}$ G. Schedelbeck, W. Wegscheider, M. Bichler, and G. Abstreiter, Science 278, 1792 (1997).

${ }^{19}$ M. Rontani, F. Troiani, U. Hohenester, and E. Molinari, Solid State Commun. 119, 309 (2001).

${ }^{20}$ A. W. Holleitner, R. H. Blick, A. K. Hüttel, K. Eberl, and J. P. Kotthaus, Science 297, 70 (2002).

${ }^{21}$ T. Ota, M. Rontani, S. Tarucha, Y. Nakata, H. Z. Song, T. Miyazawa, T. Usuki, M. Takatsu, and N. Yokoyama, Phys. Rev.
Lett. 95, 236801 (2005).

${ }^{22}$ B. Partoens and F. M. Peeters, Phys. Rev. Lett. 84, 4433 (2000); Europhys. Lett. 56, 86 (2001).

${ }^{23}$ J. J. Palacios and P. Hawrylak, Phys. Rev. B 51, 1769 (1995).

${ }^{24}$ F. Ancilotto, D. G. Austing, M. Barranco, R. Mayol, K. Muraki, M. Pi, S. Sasaki, and S. Tarucha, Phys. Rev. B 67, 205311 (2003).

${ }^{25}$ D. G. Austing, S. Tarucha, H. Tamura, K. Muraki, F. Ancilotto, M. Barranco, A. Emperador, R. Mayol, and M. Pi, Phys. Rev. B 70, 045324 (2004).

${ }^{26}$ D. Bellucci, M. Rontani, G. Goldoni, and E. Molinari, Phys. Rev. B 74, 035331 (2006).

${ }^{27}$ T. Mano, T. Kuroda, S. Sanguinetti, T. Ochiai, T. Tateno, J. Kim, T. Noda, M. Kawabe, K. Sakoda, G. Kido, and N. Koguchi, Nano Lett. 5, 425 (2005).

${ }^{28}$ T. Kuroda, T. Mano, T. Ochiai, S. Sanguinetti, K. Sakoda, G. Kido, and N. Koguchi, Phys. Rev. B 72, 205301 (2005).

${ }^{29}$ F. Suárez, D. Granados, M. L. Dotor, and J. M. García, Nanotechnology 15, S126 (2004).

${ }^{30}$ D. Granados, J. M. García, T. Ben, and S. I. Molina, Appl. Phys. Lett. 86, 071918 (2005).

${ }^{31}$ K.-H. Ahn and P. Fulde, Phys. Rev. B 62, R4813 (2000).

${ }^{32}$ Y. Li and H. M. Lu, Jpn. J. Appl. Phys., Part 1 43, 2104 (2004).

${ }^{33}$ L. G. G. V. Dias da Silva, J. M. Villas-Bôas, and S. E. Ulloa, Phys. Rev. B 76, 155306 (2007).

${ }^{34}$ B. Szafran, S. Bednarek, and M. Dudziak, Phys. Rev. B 75, 235323 (2007).

${ }^{35}$ B. Szafran, Phys. Rev. B 77, 235314 (2008).

${ }^{36}$ G. Piacente and G.-Q. Hai, J. Appl. Phys. 101, 124308 (2007).

${ }^{37}$ J. I. Climente and J. Planelles, Phys. Rev. B 72, 155322 (2005).

${ }^{38}$ L. K. Castelano, G.-Q. Hai, B. Partoens, and F. M. Peeters, Phys. Rev. B 74, 045313 (2006).

${ }^{39}$ F. Malet, M. Barranco, E. Lipparini, R. Mayol, M. Pi, J. I. Climente, and J. Planelles, Phys. Rev. B 73, 245324 (2006).

${ }^{40}$ M. Ferconi and G. Vignale, Phys. Rev. B 50, 14722 (1994).

${ }^{41}$ M. Rontani, F. Rossi, F. Manghi, and E. Molinari, Solid State Commun. 112, 151 (1999); M. Rontani, S. Amaha, K. Muraki, F. Manghi, E. Molinari, S. Tarucha, and D. G. Austing, Phys. Rev. B 69, 085327 (2004).

${ }^{42}$ S. S. Li and J. B. Xia, J. Appl. Phys. 89, 3434 (2001); A. Puente, and Ll. Serra, Phys. Rev. B 63, 125334 (2001); J. I. Climente, J. Planelles, and F. Rajadell, J. Phys.: Condens. Matter 17, 1573 (2005).

${ }^{43}$ M. Pi, A. Emperador, M. Barranco, and F. Garcias, Phys. Rev. B 63, 115316 (2001).

${ }^{44} \mathrm{~S}$. Lundqvist, in Theory of the Inhomogenous Electron Gas, edited by S. Lundqvist and N. H. March (Plenum, New York, 1983), p. 149.

${ }^{45}$ J. P. Perdew and A. Zunger, Phys. Rev. B 23, 5048 (1981). 